\title{
Review
}

\section{The Study of Hepatitis B Virus Reactivation}

\author{
Zhao-chun Chi
}

Hepatitis B virus (HBV) reactivation after chemotherapy or immunosuppressive therapy is a cause of liver-related morbidity and mortality. Not all chronic hepatitis B patients will lead to HBV reactivation. The incidence is $0.3 \%$ $30.2 \%$ according to the reports. The mechanism of HBV reactivation is still unclear, but it is believed that the viral load is increasing due to the suppression of immune response. No uniform diagnostic criteria are available. HBV reactivation can be confirmed by an increase of serum HBV DNA level. Recently, awareness of reactivation of occult HBV has been improved, especially in HBV endemic area. Preemptive antiviral therapy was the best approach to prevent the HBV reactivation. HBV reactivation can lead to acute hepatitis, severe hepatitis and acute liver failure. Therefore, it is worthy of great attention and further study. Antiviral therapy is safe and effective to prevent HBV reactivation.

Key words: HBV reactivation; Occult HBV infection; Chemotherapy; Hepatectomy; Immunosuppressive

$\mathrm{H}$ $\mathrm{BV}$ reactivation refers to the previous history of HBV infection has been deactivated or recovered under some circumstances, such as chemotherapy, immunosuppression therapy, liver resection under the condition of serum HBV DNA is increasing, always accompanied by hepatitis flare. Elevated transaminase levels and clinical manifestations of acute hepatitis may cause acute liver failure, and even death. HBV reactivation usually uses HBV DNA level as diagnostic criteria, namely HBV DNA $>10^{3} \mathrm{IU} /$ $\mathrm{ml}$, or 2 consecutive detection of hepatitis B surface antigen (HBsAg) positive caused by chemotherapy, immunosuppression therapy, operation and other factors $^{1-3}$ in patients with undetectable HBV DNA or HBsAg.

Generally, the inactivation rate of $\mathrm{HBV}$ after the HBV reactivation is quite low. Tohme et al ${ }^{4}$ followed up 414 patients in the inactive phase of hepatitis B (HBV DNA < $2000 \mathrm{IU} / \mathrm{ml}$, and normal alanine aminotransferase for 12 months) for 10 years. A total of $36(9 \%)$ persons had reactivation with an annual incidence of $1.2 \%$. Risk factors for $\mathrm{HBV}$ reactivation were male with $\mathrm{HBV}$ DNA $\geqslant 1000 \mathrm{IU} / \mathrm{ml}$, and $\mathrm{HBV}$

Department of Gastroenterology, Qingdao Municipal Hospital, Affiliated Hospital of College Pharmacy of Ocean University of China Correspondence: Zhao-chun Chi, Email: c.z.chow@163.com genotype $\mathrm{B}$. They suggested that males and those with HBV DNA $\geqslant 1000 \mathrm{IU} / \mathrm{ml}$ need to be followed up.

Under circumstances of tumor, autoimmune diseases, malignant blood disease, rheumatism and other diseases, the immune inhibitor or chemotherapy is the most effective treatment method. However, the patients with negative original HBV infection, i.e. HBsAg-negative and antibodies to hepatitis B core antigen (anti-HBc) positive patients can develop HBV reactivation even 2 years after stopping immunosuppression. ${ }^{1,5,6}$

In the process of immune therapy or chemotherapy, immune function is suppressed, which results in HBV replication in vivo and infecting more liver cells. HBV DNA has response element of glucocorticoid. Glucocorticoid can activate HBV gene expression and induce HBV replication. Rituximab may lead to mature B cells and plasma cells decreasing, resulting in antibody production decline, causing humoral immune dysfunction, thus inducing HBV reactivation. ${ }^{2,7,8}$

Liver injury induced by HBV reactivation can occur after stopping drug or in the process of medication. Because a great quantity replication of HBV in liver cells, and attack from cytotoxic 
$\mathrm{T}$ cells to liver cells infected by HBV, can cause inflammation, necrosis and apoptosis, eventually lead to fibrosis.

\section{Occult $\mathrm{HBV}$ infection and $\mathrm{HBV}$ reactivation}

Occult HBV infection (OBI) is defined as presence of HBV DNA in the liver tissue of patients with serologically undetectable HBsAg. Majority of OBI are positive for anti-HBs and/or anti-HBc and minor portion are negative for all HBV markers. The study found that HBV persists in the presence of covalently closed circular DNA (cccDNA). OBI increases the risk of $\mathrm{HBV}$ transmission through transfusion, hemodialysis and organ transplantation. Therefore, in order to patient's safety, the OBI should be determined and effectively screened. Antiviral therapy is needed in HBsAg-negative transplant. Patients who are anti-HBc positive can prevent the recurrence of $\mathrm{HBV}$ infection through it. Since HBV replication can be suppressed by immune surveillance system in OBI patients. Immunosuppression results in massive HBV replication. This leads to acute hepatitis and sometimes mortality when immune surveillance is recovered after stopping immunosuppressive drugs/ anticancer chemotherapy., ${ }^{9,10}$

Occult HBV infection may occur in subject with solid tumours, although the risk of its reactivation under chemotherapy appears to be very low. ${ }^{10}$ Chen et $\mathrm{al}^{11}$ retrospectively analyzed 322 occult HBV carriers who received kidney transplantation, and found 15 cases $(4.7 \%)$ had HBV reactivation. Older age and anti-T-cell antibodies may increase the risk of HBV reactivation, whereas lamivudine prophylaxis may prevent HBV reactivation after kidney transplantation.

OBI may have impacted on several different clinical contexts. In fact, it can be transmitted through blood transfusion and liver transplantation causing classic forms of hepatitis B in newly infected individuals. The process of an immunotherapy or chemotherapy may induce OBI reactivation and develop to acute and severe hepatitis. OBI can favor the progression of liver fibrosis. The possible evidence suggests that the effect of OBI to the establishment of cirrhosis also implies its possible indirect role in the development of hepatocellular carcinoma. ${ }^{10}$

Individuals negative for $\mathrm{HBsAg}$ but positive for anti-HBc are at risk of $\mathrm{HBV}$ reactivation under immunosuppressive conditions. Inuzuka et al ${ }^{12}$ examined the prevalence of the G1896A variant in 14 individuals originally HBsAg-negative but anti$\mathrm{HBc}$-positive that experienced $\mathrm{HBV}$ reactivation and 44 healthy individuals that were $\mathrm{HBsAg}$-negative but anti-HBc-positive without HBV reactivation, and found that the G1896A variant was detected in $42.9 \%$ (6/14) of cases, including two cases with fatal liver failure, while it was observed in the liver tissue of $11.4 \%(5 / 44)$ of indivividuals with OBI. They thought that reactivation from OBI was characterized by low genetic heterogeneity, with the wild-type G1896 or G1896A variant prevalent.

In a word, OBI may be involved in different clinical contexts, including the transmission of the infection by blood transfusion, liver transplantation and its acute reactivation when an immunosuppressive status occurs. Moreover, much evidence suggests that it may contribute to the development of cirrhosis and have an important role in hepatocarcinogenesis. ${ }^{13}$

\section{The incidence and risk factors}

The incidence of HBV reactivation according to the different reports is $0.3 \%-30.2 \%$. The occurrence of HBV reactivation is associated with virus infection status, drugs, suffering kinds of disease and other related factors. Among them, suffering kinds of disease and drug have the most impact. Use of immunosuppressive drugs before the HBsAg $(+), \mathrm{HBeAg}(+), \mathrm{HBV}$ DNA $>2000 \mathrm{IU} / \mathrm{ml}$ is a risk factor for HBV reactivation. Chemotherapy package, corticosteroid, monoclonal antibody and anthracycline antibiotics can significantly increase HBV reactivation. Patients with age $>50$ years old have obviously high HBV reactivation rate and they still have other dangerous malignant hematological diseases. HBsAg negative/HBcAb positive haematopoietic stem cell transplant (HSCT) recipients are at high risk of $\mathrm{HBV}$ reactivation ${ }^{13-15}$

\section{Immunosuppression and $\mathrm{HBV}$ reactivation}

Immunosuppressive drugs associated with HBV reactivation contain corticosteroids primarily (dexamethasone, prednisone, prednisolone) and 
monoclonal antibodies (rituximab, tocilizumab, etanercept).

With the increasing use of immunosuppressive therapy, HBV reactivation in endemic regions becomes a clinical problem requiring special attention. A recent survey clarified that HBV reactivation related to immunosuppressive therapy had been increasing in patients with malignant lymphoma, other hematological malignancies, oncological or rheumatoid diseases. Rituximab plus steroid-containing chemotherapy was identified as a risk factor for $\mathrm{HBV}$ reactivation in $\mathrm{HBsAg}$ negative patients with malignant lymphoma. ${ }^{16}$ The use of tumor necrosis factor- $\alpha$ (TNF- $\alpha$ ) inhibitors is increasing especially in patients with rheumatoid arthritis (RA). As TNF- $\alpha$ inhibitors are strongly immunosuppressive, the occurrence of HBV reactivation has been recently observed. Reports suggest a higher risk of complicating HBV reactivation in carriers who are treated with TNF- $\alpha$ inhibitors. Therefore, HBV carriers are recommended to undergo prophylactic administration of nucleotide analogs (NAs). Tanaka et $\mathrm{al}^{17}$ uncovered several characteristics of the de novo hepatitis B due to TNF- $\alpha$ inhibitors. First, the time between the start of TNF- $\alpha$ inhibitors and the occurrence of de novo hepatitis was longer than one year. Second, patients were usually treated with additional non-biologic agents, which also had immunosuppressive effects. Third, the disease could be fatal. Fourth, several types of TNF- $\alpha$ inhibitors exhibited a risk of developing de novo hepatitis. The incidence of de novo hepatitis B is $0-5 \% /$ year.

$\mathrm{HBV}$ reactivation rate of patients with rheumatic disease receiving prednisone is $20.8 \%$ compared to only $4.46 \%$ and $9.52 \%$ of patients treated with DMARDs (disease-modifying anti-rheumatic drugs) or TNFBA (TNF- $\alpha$-blocking agents), respectively. This long-term (16-48 months) follow-up suggests that pre-emptive antiviral therapy should be administered in patients receiving prednisone therapy for rheumatic disease. ${ }^{18}$

\section{Tumor chemotherapy and $\mathrm{HBV}$ reactivation}

HBV reactivation may occur with chemotherapy and has significant morbidity and mortality. The United
States Centre for Disease Control and Prevention recommends prechemotherapy hepatitis B screening for all cancer patients, while American Society of Clinical Oncology finds that there is insufficient evidence currently to support such a recommendation. Ling et al ${ }^{19}$ reported 1149 tumor patients, including 434 with doxorubicin-based, 196 with oxaliplatinoririnotecan-based, 245 with carboplatin/gemcitabine and 274 with capecitabine chemotherapy, and HBV screening rate was 39\% (448/1 149) overall. About 7\% (30/448) screened patients were HBsAg positive and 28 out of 30 received prophylactic antiviral therapy with no HBV reactivation. Only $0.3 \%(3 / 1149)$ patients developed HBV reactivation. Actually $1.4 \%$ (3/214) from the unscreened doxorubicin group and $0 \%(0 / 487)$ from no unscreened patients in the other three treatment groups developed reactivation $(P<$ 0.001). Not all chemotherapy regimens lead to HBV reactivation. Routine hepatitis B screening for lowor moderate-risk regimens may not be warranted. Prophylactic administration of lamivudine to lymphoma patients infected with HBV may reduce the morbidity and mortality associated with HBV reactivation. ${ }^{20}$

A total of 109 patients with malignant lymphoma or multiple myeloma are in one study. They were checked anti-HBs or anti-HBc before treatment, and HBV DNA in sera was quantified monthly during and after chemotherapy. Out of 109 patients, 42 (38.5\%) had anti-HBs and 59 (54.1\%) had anti-HBc. Among the 59 anti-HBc positive patients, $6.8 \%$ (4/59) showed HBV reactivation. ${ }^{2}$ The rate of $\mathrm{HBV}$ reactivation is low in patients who were HBsAg negative/anti$\mathrm{HBc}$ positive receiving rituximab-based combination chemotherapy without concomitant antiviral prophylaxis. But in the elderly patients, particularly those without anti-HBs seemed particularly at risk. ${ }^{21}$

\section{Hematopoietic stem cell transplantation and HBV reactivation}

HBsAg-negative/HBcAb-positive haematopoietic stem cell transplant (HSCT) recipients are at high risk of $\mathrm{HBV}$ reactivation. Mikulska et $\mathrm{al}^{15}$ studied the impact of being $\mathrm{HBsAg-negative/HBcAb-positive} \mathrm{in}$ this population. Overall, 137 of 764 patients (18\%) 
were $\mathrm{HBs} A g$-negative/HBcAb-positive before HSCT. Overall survival, non relapse mortality (NRM), acute and chronic graft vs. host disease were similar in $\mathrm{HBcAb}$-positive and $\mathrm{HBcAb}$-negative patients. Reactivation occurred in 14 patients $(10 \%)$ within a median of 19 months after HSCT (range 9-77). Cause-specific hazard for reactivation was increased in patients who received rituximab treatment. No differences in overall survival and NRM were found between patients with and without HBV reactivation. The donor's immunity was independently and consistently associated with a decreased risk of HBV reactivation.

Prophylaxis with lamivudine is recommended for $\mathrm{HBcAb}$-positive allogenic hematopoietic stem cell transplant (HSCT) recipient, but the optimal timing for the institution and duration of the prophylaxis is still unknown. In addition, considering the high rate of mortality associated with HBV reactivation, the most potent and long-term effective antiviral regimen should be considered. Milazzo et al ${ }^{22}$ reported a case of late onset of HBV reactivation after a longterm prophylaxis with prophylaxis with lamivudine in a patient who underwent HSCT for non-Hodgkin lymphoma and who was successfully treated with a combination antiviral regimen including entecavir and tenofovir disoproxil fumarate.

\section{Radio frequency ablation or hepatic resection and HBV reactivation}

Radio frequency ablation (RFA) is a local ablation therapy for hepatocellular carcinoma in the application of the most extensive ablation technique. The incidence of $\mathrm{HBV}$ reactivation was lower in patients who received liver resection to treat HBV-related hepatocellular carcinoma. Antiviral therapy prior to the hepatic resection treatment may be beneficial for reducing the incidence of HBV reactivation. A study found that the incidence was significantly lower in the RFA group than in the liver resection group $(5.6 \% \mathrm{vs}$. $14.0 \%, P=0.034)$. The univariate and multivariate analysis indicated that the treatment type were significant risk factors of $\mathrm{HBV}$ reactivation. However, the incidence of $\mathrm{HBV}$ reactivation in patients who did not receive antiviral therapy was higher than in those who did receive antiviral therapy in the liver resection group $(12 / 58$ vs. $1 / 35) .^{23}$

Dan et $\mathrm{al}^{24}$ studied 218 consecutive patients occur with HBV-related small HCC treated with RFA $(\mathrm{n}=125)$ or liver resection $(\mathrm{n}=93)$. HBV reactivation developed in $20(9.2 \%)$ patients after treatment. The incidence of HBV reactivation was also greatly lower in the RFA group $(5.6 \%, 7 / 125)$ than the liver resection group $(14.0 \%, 13 / 93)$. Treatment with RFA/liver resection was significant risk factor of HBV reactivation. About subgroup analysis, the incidence of HBV reactivation was lower in patients who received antiviral therapy than those who did not receive antiviral therapy. Therefore prophylactic antiviral therapy is recommended.

\section{Rheumatoid arthritis therapy and HBV reactivation}

Nakamura et $\mathrm{al}^{25}$ studied the incidence of HBV reactivation of 244 rheumatoid arthritis (RA) patients with anti-HBc and/or anti-HBs positive receiving biological disease-modifying antirheumatic drugs (DMARDs). HBV reactivation occurrent in 5.3\% of RA patients with prior HBV infection during treatment with biological DMARDs, but there were no associated clinical manifestations. Accordingly, it seems that biological DMARDs can be used safely in patients with RA. Xuan et al ${ }^{18}$ studied 12 patients received prednisone, DMARDs or TNFBA, HBV reactivation was only documented in two patients with prednisone without pre-emptive antiviral therapy. One hundred patients from literature review were identified as having HBV reactivation. About $20.8 \%$ of the patients receiving prednisone compared to only $4.46 \%$ and $9.52 \%$ of the patients treated with DMARDs or TNFBA, respectively.

Juvenile rheumatoid arthritis is a common chronic inflammatory disease. Some of the drugs used for the treatment have immunosuppressive activity. One of the serious side-effects of immunosuppressive treatment is activation of opportunistic pathogens. $\mathrm{HBV}$ is one of these pathogens. HBV reactivation can cause liver damage and death, so antiviral therapy is recommended. ${ }^{26}$ 
Tamori et $\mathrm{al}^{27}$ enrolled 50 patients with RA who had anti-HBc and who had started treatment with DMARDs, including who had received antiTNF $\alpha$. HBV reactivation occurred in 2 of 5 patients with HBsAg and in 1 of 45 patients without HBsAg. The inciedence of HBV reactivation was low in RA. Prophylactic therapy with entecavir were effective to prevent $\mathrm{HBV}$-associated liver failure in patients with HBsAg.

\section{Liver transplantation in tumor patients with $\mathrm{HBV}$ reactivation}

Acute on chronic liver failure (ACLF) occurs in tumor patients because of HBV reactivation. Kim et $\mathrm{al}^{28}$ studied twenty patients who underwent orthotopic liver transplantation (OLT) for ACLF due to HBV reactivation. Study revealed that OLT is a feasible and effective approach in ACLF due to HBV reactivation in select lymphoma patients.

\section{Prevention of $\mathrm{HBV}$ reactivation}

At present, whether the HBsAg (-), HBcAb $(+)$ need prophylactic antiviral therapy is still controversial. "China Guidelines for the Prevention and Treatment of Chronic Hepatitis B (2010)" clearly pointed out that the HBsAg (-) and $\mathrm{HBcAb}$ $(+)$ patients who acceptted long-term or high-dose immunosuppressive or cytotoxic drugs should be closely monitored the HBV DNA and HBsAg. If positive, it should be combined with antiviral drug. For patients with baseline HBV DNA $<2000 \mathrm{IU} /$ $\mathrm{ml}$, it should continue therapy for 6 months after completing chemotherapy or immunosuppressive therapy. First, such patients especially occult HBV infection patients should be routinely performed HBV monitorings. HBV DNA and liver function should be closely monitored during treatment. All patients should be screened for HBsAg by a sensitive method before immunosuppressive therapy. Second, $\mathrm{HBcAb}$ and $\mathrm{HBsAb}$ testing should be performed in HBsAg negative patients, especially those receiving intensive immunosuppressive therapy. Prophylaxis with nucleoside analog is essential for preventing HBV reactivation in HBsAg positive patients. In contrast, $\mathrm{HBsAg}$ negative with $\mathrm{HBcAb}$ and/or HBsAb positivce patients should be monitored HBV DNA in serum monthly for 12 months after completion of chemotherapy. Nucleoside analogs should be administrated immediately when HBV DNA becomes positive during this period. This strategy facilitates commencement of nucleoside analogs at an early stage of HBV reactivation and results in prevention of severe hepatitis. The antiviral prophylaxis prior to chemotherapy and its continuation until reconstitution of normal host immunity is the cornerstone for effective prevention of HBV reactivation. ${ }^{16,29,30} \mathrm{~A}$ antiviral therapy decreases the risk of perioperative viral reactivation in patients with $\mathrm{HBV}$-induced hepatocellular carcinoma. Among the 84 patients with HBV-induced hepatocellular carcinoma, 15 patients (17.8\%) developed HBV reactivation during the perioperative period, of which $8(57.1 \%)$ were within the first week after hepatoctomy. The incidence of $\mathrm{HBV}$ reactivation during the perioperative period was $2.5 \%(1 / 40)$ in the antiviral-treated group compared with $31.8 \%(14 / 44)$ in the control group. ${ }^{31}$ Antiviral therapy with telbivudine can significantly decrease the perioperative $\mathrm{HBV}$ reactivation in patients with HBV-related hepatocellular carcinoma undergoing liver resection. ${ }^{30}$

In rituximab-containing chemotherapy for B-cell lymphoma, severe hepatitis due to HBV reactivation occured. Wang et $\mathrm{al}^{32}$ studied 268 patients with B-cell lymphoma who received rituximab-containing chemotherapy. Results supported that prophylactic lamivudine could prevent HBV reactivation for B-cell lymphoma with HBV infection who was receiving rituximab-containing chemotherapy. ${ }^{32}$

In summary, HBV associated with hepatocellular carcinoma, B cell lymphoma underwent with monoclonal antibody chemotherapy, radiotherapy, operation, rheumatoid arthritis receiving immunosuppressive therapy, allogeneic hematopoietic stem cell transplantation and liver transplantation should be closely monitored HBsAg. HBV DNA can early detect $\mathrm{HBV}$ reactivation. In order to prevent HBV reactivation, consensus has been forged that antiviral treatment is an effective and safe approach. 


\section{REFERENCES}

1. Zachou K, Sarantopoulos A, Gatselis NK, Vassiliadis T, Gabeta S, Stefos A, et al. Hepatitis B virus reactivation in hepatitis B virus surface antigen negative patients receiving immunosuppression: A hidden threat. World J Hepatol 2013;5(7):387-392.

2. Matsui T, Kang JH, Nojima M, Tomonari A, Aoki H, Yamazaki $\mathrm{H}$, et al. Reactivation of hepatitis B virus in patients with undetectable HBsAg undergoing chemotherapy for malignant lymphoma or multiple myeloma. J Med Virol 2013;85(11):19001906.

3. Huang YH, Hsiao LT, Hong YC, Chiou TJ, Yu YB, Gau JP, et al. Randomized controlled trial of entecavir prophylaxis for rituximab-associated hepatitis B virus reactivation in patients with lymphoma and resolved hepatitis B. J Clin Oncol 2013;31(22):2765-2772.

4. Tohme RA, Bulkow L, Homan CE, Negus S, McMahon BJ. Rates and risk factors for hepatitis $B$ reactivation in a cohort of persons in the inactive phase of chronic hepatitis B-Alaska, 2001-2010. J Clin Virol 2013;58(2):396-400.

5. Shouva D, Shibolet O. Immunosuppression and HBV reactivation. Semin Liver Dis 2013;33(2):167-177.

6. Hsu C, Tsou HH, Lin SJ, Wang MC, Yao M, Hwang WL. Chemotherapy-induced hepatitis $\mathrm{B}$ reactivation in lymphoma patients with resolved HBV infection: A prospective study. Hepatology 2014;59(6):2092-2100.

7. Oh MJ, Lee HJ. A study of hepatitis B virus reactivation associated with rituximab therapy in realworld clinical practice: a single center experience. J Clin Mol Hepatol 2013;19(1):51-59.

8. Elkady A, Aboulfotuh S, Ali EM, Sayed D, Abdel-Aziz NM, Ali $\mathrm{AM}$, et al. Incidence and characteristics of HBV reactivation in hematological malignant patients in south Egypt. World J Gastroenterol 2013;19(37):6214-6220.

9. Song SH, Hwang SG. Occult hepatitis B virus infection: transmission and reactivation. Korean $\mathrm{J}$ Gastroenterol 2013;62(3):148-153.

10. Raimondo G, Caccamo G, Filomis R, Pollicino T. Occult HBV infection. Semin Immunopathol 2013;35(1):39-52.

11. Chen GD, Gu JL, Qiu J, Chen LZ. Outcome and risk factors for hepatitis B virus (HBV) reactivation after kidney transplantation in occult HBV carriers. Transpl Infect Dis 2013;15(3):300-305.

12. Inuzuka T, Ueda $Y$, Morimura $H$, Fujii $Y$, Umeda M, Kou T. Reactivation from occult HBV carrier status is characterized by low genetic heterogeneity with the wild-type or G1896A variant prevalence. J Hepatol 2014;61(3):492-501.

13. Squadrito G, Spinella R, Raimondo G. The clinical significance of occult HBV infection. Ann Gastroenterol 2014;27(1):15-19.

14. Goossens N, Negro F. Reactivation of hepatitis B associated with immunosuppression. Rev Med Suisse 2013;9(369):1566-1568.

15. Mikulska M, Nicolini L, Signori A, Rivoli G, Del Bono V, Raiola AM, et al. Hepatitis B reactivation in HBsAg-negative/ $\mathrm{HBcAb}$-positive allogeneic haematopoietic stem cell transplant recipients: risk factors and outcome. Clin Microbiol Infect 2014; doi: 10.1111/1469-0691.12611

16. Oketani M, Ido A, Uto H, Tsubouchi H. Prevention of hepatitis $\mathrm{B}$ virus reactivation in patients receiving immunosuppressive therapy or chemotherapy. Hepatol Res 2012;42(7):627-636.

17. Tanaka E, Urata Y. Risk of hepatitis B reactivation in patients treated with tumor necrosis factor- $\alpha$ inhibitors. Hepatol Res 2012;42(4):333-339.

18. Xuan D, Yu Y, Shao L, Wang J, Zhang W, Zou H. Hepatitis reactivation in patients with rheumatic diseases after immunosuppressive therapy-a report of long-term followup of serial cases and literature review. Clin Rheumatol 2014;33(4):577-586.

19. Ling WH, Soe PP, Pang AS, Lee SC. Hepatitis B virus reactivation risk varies with chemotherapy regimens commonly in solid tumours. Br J Cancer 2013;108(10):1931-1935.

20. Liu WP, Zheng W, Song YQ, Ping LY, Wang GQ, Zhu J. Hepatitis B surface antigen seroconversion after HBV reactivation in nonHodgkin's lymphoma. World J Gastroenterol 2014;20(17):51655170

21. Koo YX, Tay M, Teh YE, Teng D, Tan DS, Tan IB, et al. Risk of hepatitis B virus (HBV) reactivation in hepatitis B surface antigen negative/hepatitis B core antibody positive patients receiving rituximab-containing combination chemotherapy without routine antiviral prophylaxis. Ann Hematol 201;90(10):1219-1223.

22. Milazzo L, Corbellino M, Foschi A, Micheli V, Dodero A, Mazzocchi A, et al. Late onset of hepatitis B virus treatment fllowing hematopoietic stem cell transplantation: successful treatment with combined entecavir plus tenofovir therapy. Transpl Infect Dis 2012;14(1):95-98

23. Liu F, Dan J, Zhang Y. Hepatitis B virus reactivation after treatment for HBV-related hepatocellular carcinoma: comparative analysis of radiofrequency ablation versus hepatic resection. Zhonghua Gan Zang Bing Za Zhi (chin) 2014;22(1):38-42.

24. Dan JQ, Zhang YJ, Huang JT, Chen MS, Gao HJ, Peng ZW, et al. Hepatitis B virus reactivation radiofrequency ablation or hepatic resection for HBV-related small hepatocellular carcinoma: a retrospective study. Eur J Surg Oncol 2013;39(8):865-872.

25. Nakamura J, Nagashima T, Nagatani K, Yoshio T, Iwamoto M, Minota S. Reactivation of hepatitis B virus in rheumatoid arthritis patients treated with biological disease-modifying antirheumatic drugs. Int J Rheum Dis 2014;doi:10.1111/1756-185x.12359.

26. Durmus O, Tekin L, Carli E, Cakar E, Acar A, Ulcay A. Hepatitis $\mathrm{B}$ virus reactivation in a juvenile rheumatoid arthritis patients under treatment and its successful management:a complicated case. Rheumatol Int 2013;33(5):1345-1349.

27. Tamon A, Koike T, Goto H, Wakitani S, Tada M, Morikawa $\mathrm{H}$, et al. Prospective study of reactivation of hepatitis B virus in patients with rheumatoid arthritis who received immunosuppressive therapy: evaluation of both HBsAg-positive and HBsAg-negative cohorts. J Gastroenterol 2011;46(4):556564.

28. Kim JM, Kwon CH, Joh JW, Ko SW, Park JB, Kim SJ, et al. Liver transplantation in lymphoma patients with hepatitis B virus reactivation. Transplant Proc 2013;45(8):2988-2991.

29. Ohishi W, Chayama K. Prevention of hepatitis B reactivation in immunosuppressive therapy or chemotherapy. Clin Exp Nephrol 2011;15(5):634-640.

30. Lubel JS, Angus PW. Hepatitis B reactivation in patients receiving cytotoxic chemotherapy: diagnosis and management. J Gastroenterol Hepoatol 2010;25(5):664-671.

31. Huang L, Li J, Yan J, Sun J, Zhang X, Wu M. Antiviral therapy decreases viral reactivation in patients with hepatitis B virusrelated hepatocellular carcinoma undergoing hepatectomy: a randomized controlled trial. J Viral Hepal 2013;20(5):336-342.

32. Wang YH, Fan L, Wang L, Zhang R, Xu J, Fang C, et al. Effect of prophylactic lamivudine to prevent hepatitis $\mathrm{B}$ virus reactivation in B-cell lymphoma treated with rituximabcontaining chemotherapy. Support Care Cancer 2013;21(5): $1265-1271$ 